\title{
Theoretical analysis of structural diversity of covalent organic framework: Stacking isomer structures thermodynamics and kinetics
}

\author{
Taku Hayashi ${ }^{\mathrm{a}}$,Yuh Hijikata * a, b Alister Page $^{\mathrm{c}}$, Donglin Jiang $^{\mathrm{d}}$, and Stephan Irle ${ }^{*}$, a, b \\ ${ }^{a}$ Department of Chemistry Graduate School of Science, Nagoya University, Chikusa-ku, \\ Nagoya, 464-8602, Japan \\ ${ }^{\mathrm{b}}$ Institute of Transformative Bio-Molecules (WPI-ITbM), Nagoya University, Chikusa-ku, \\ Nagoya, 464-8602, Japan \\ ${ }^{\mathrm{c} D i s c i p l i n e}$ of Chemistry, School of Environmental and Life Science, the University of \\ Newcastle, Callaghan 2308, Australia \\ ${ }^{\mathrm{d}}$ Field of Energy and Environment, School of Materials Science, Japan Advanced Institute of \\ Science and Technology, 1-1 Asahidai, Nomi 923-1292, Japan
}

* E-Mail: hijikata@itbm.nagoya-u.ac.jp (Y.H.), sirle@,chem.nagoya-u.ac.jp (S.I.) 


\begin{abstract}
Covalent organic frameworks (COFs) have attracted much interest due to their utility as functional materials. Unfortunately, experimental synthesis struggles with low single crystallinity of COFs. We have theoretically investigated isomer structures of a representative two-dimensional $\mathrm{COF}$ for both monolayer and three-dimensional stacking orders. We show that rotations of $p$-phenylene rings are common in monolayers, however, affect the global stacking order substantially. We also discuss the discrepancy between powder X-ray diffraction patterns corresponding to the structures predicted by our calculations and those experimentally observed. The discrepancy demonstrates the importance of dynamics in the self-assembly process of COF organic components.
\end{abstract}




\section{Introduction}

Porous materials are highly attractive for many researchers because of their applicability in diverse areas such as gas storage and catalysis [1,2]. One class of such fascinating crystalline porous materials are porous coordination polymers (PCPs) / metal organic frameworks (MOFs) which have advantages in designability of framework structures and tunability of pore size to perform various functions [2]. Another class of porous crystalline materials called covalent organic frameworks (COFs) emerged in 2005 [3]. The COFs are often two-dimensional (2D) organic co-polymers where at least two different types of monomer units are connected via covalent bonds to produce large surface sheets which can be stacked on top of each other via $\pi-\pi$ interactions. The columns then form pores whose sizes are tunable via the choice of the monomer units. COFs exhibit low densities and high thermal stabilities, similar to MOFs [4-6]. They have been shown to perform various functions [3, 7-11], which are affected not only by the choice of the monomer units, but also by the global structure of the framework [12-15]. Unfortunately, structural characterization of COFs is not straightforward due to the general difficulties in producing single crystals of sufficiently large size, which is related to their bond formation processes that exhibit lower reversibility compared with other, more crystalline porous materials such as PCPs/MOFs where coordination bonds are typically formed in equilibrium reactions [5, 16-19]. Rietveld analysis is usually used in the case of COFs to reveal their structures from powder X-ray diffraction (XRD) patterns [20,21]. Construction of initial model structures is crucial in the procedures 
analyzing these patterns, and the difficulty of such construction is also well known [22]. On the other hand, theoretical prediction of possible structures has become a practical approach because quantum chemical methods such as density functional theory (DFT) are capable of exploring various candidate structures. Thus, theoretical calculations can predict their relative energies and on occasion also the local dynamics of the frameworks, which may also exert effects on the three-dimensional (3D) structures and functions [23]. For such elaborate investigations it is advisable to employ computationally more efficient, approximate DFT methods such as density-functional tight-binding (DFTB).

In this work, we theoretically investigated the geometries, energetics and dynamic properties of various stacking orders of a particular double-stage COF recently synthesized by Jiang et al. [24]. It is constructed from zinc 5, 10, 15, 20-tetrakis(4'-tetraphenylamino) porphyrin (ZnP), 2, 3, 9, 10, 16, 17, 23, 24-octahydroxyphthalocyaninato copper (II) (CuPc) and 4-formylphenylboronic acid (FPBA) under solvothermal conditions. In this reaction, boronic ester is formed between $\mathrm{CuPc}$ and FPBA, and imine is formed between $\mathrm{ZnP}$ and FPBA to build the rectangular COF as shown in Fig. 1. Hereafter, we call this COF CuPc-FPBA-ZnP. We selected this system because it exhibits a wide variety of monolayer structures due to it being a three component system, and formally freely rotatable $p$-phenylene groups that can interfere with the stacking order as explained below. We considered two representative isomers of the CuPc-FPBA-ZnP monolayer, one is square (S) and has 
tetragonal pores, while the other is rhombic $(\mathbf{R})$ and has rhombic pores. In the $\mathbf{S}$ isomer, the

four imine nitrogens around the $\mathrm{ZnP}$ units are connected to the $p$-phenylene groups of FPBA forming identical dihedral angles, whereas in the $\mathbf{R}$ structure these dihedral angles alternate by $180^{\circ}$ for nitrogens connected to nearest-neighbored FPBA units. We evaluate the layer stabilization energies in various stacking orders of $\mathbf{S}$ and $\mathbf{R}$ monolayers, and compare theoretically predicted XRD patterns with the experimental data for this COF. To shed insight into the dynamic properties of the $p$-phenylene groups connected to the imine nitrogens, we investigated the influence of their rotation in one of the optimized 3D structures.

\section{Computational details}

\subsection{Quantum chemical calculations}

The optimization of the monolayers and their layered structures were performed using the self-consistent charge density-functional tight-binding (SCC-DFTB, nowadays often referred to as DFTB2) method as implemented in the DFTB+ program package version 1.2.2 [25]. DFTB employs a valence-only two-center approximation with a minimal basis set, and has parameterized Hamiltonian and overlap matrix elements, hence is faster than DFT by up to three orders of magnitude. Lennard-Jones type dispersion with the universal force field (UFF) parameters was included in all calculations to include $\pi$-stacking interactions [26]. Further details on the construction of the DFTB Hamiltonian are explained in the supporting 
information (SI). From now we refer to SCC-DFTB including UFF dispersion as "DFTB" for brevity. Single point DFT energies with the PBE exchange functional [27] and the second version of Grimme's dispersion correction [28] were performed to validate the computed DFTB energies. Details of the DFT calculations are given in the SI as well.

The optimization of atomic geometries and all three lattice vectors were conducted in periodic boundary conditions $(\mathrm{PBC})$ using the $\Gamma$-point approximation. The conjugate gradient algorithm was employed in geometry optimizations using $1 \times 10^{-3} \mathrm{Ha} / \mathrm{a}_{0}$ as a force threshold. We employed $100 \AA$ and $4.5 \AA$ as initial distances separating adjacent 2D monolayers to optimize individual monolayer geometries and stacked structures, respectively.

\subsection{Design of monolayer models}

Unit cells of $\mathbf{S}$ and $\mathbf{R}$ isomers are shown in Fig. S1 in the SI. In each isomer, there can exist four distinct rotational conformers related to the rotation of $p$-phenylene groups around $\mathrm{ZnP}$, which should twist out of the plane due to steric hindrance between their ortho-hydrogen and the closest hydrogen atoms of the $\mathrm{ZnP}$ unit (see Fig. 2). We labeled these local isomers $C_{2}, C_{4}, S_{4}$, and $C_{2} \mathrm{xy}$, based on their local symmetries. We optimized the geometries of these eight monolayer structures and labeled them individually, for instance the $\mathrm{S}$ isomer with $C_{2}$ conformation was named $\mathbf{S} \_C_{2}$. 


\subsection{Design and characterization of stacked structure models}

Within one unit cell we stacked two layers of the four optimized monolayers for each $\mathbf{R}$ and $\mathbf{S}$ forms as initial structures as shown in Fig. 3. For the $\mathbf{R}$ isomer, we considered eight different types of vertical stacking order, namely eclipsed (E), staggered (SG), serrated $\mathrm{x}$ directon (SR-x), serrated y direction (SR-y), serrated xy direction (SR-xy), inclined $\mathrm{x}$ direction (I-x), inclined y direction (I-y), and inclined xy direction (I-xy) types. Ref. 13 gives a good account of the importance of the stacking order in COFs. $\quad \mathbf{E}$ denotes perfectly eclipsed vertical stacking. SG is a staggered structure in which a vertex of one layer is situated below the center of the pore of the next layer. SR-x, SR-y and SR-xy are repetitive structures in which the second layer slips slightly in the $\mathrm{x}, \mathrm{y}$ and $\mathrm{xy}$ directions from $\mathbf{E}$, respectively, and the third layer (which is in the periodic image of the unit cell) is perfectly eclipsed with the first layer. Thus, the unit cell angles $\alpha$ and $\beta$, measuring the direction of the unit cell vector associated with vertical stacking, are $90^{\circ}$ in these structures. I-x, I-y and I-xy are slipped structures in $\mathrm{x}, \mathrm{y}$ and $\mathrm{xy}$ directions, respectively, but here the third layer is also slipped by the same angle with respect to the second layer as the second layer is with respect to the first. Hence, in inclined $\mathbf{I}$ structures, $\alpha, \beta<90^{\circ}$. In the case of the $\mathbf{S}$ isomers, SR-y and I-y can be omitted because $\mathrm{x}$ and y directions are equivalent. The initial slipping distance in $\mathbf{S R}$ and $\mathbf{I}$ is $1.0,1.5$ and $2.0 \AA$ for the $\mathbf{S}$ form and $1.5 \AA$ for the $\mathbf{R}$ form, as will be discussed later. Thus, we considered a total of 56 different stacked structures for the $\mathbf{S}$ 
isomers and 32 different stacked structures for the $\mathbf{R}$ isomers as initial structures for the geometry optimization. We placed two layers in one unit cell for all the layered structures to investigate their stacking order. The powder XRD patterns of each optimized structure were simulated using the Mercury 3.3.1 code, using $\lambda=1.54 \AA$ [29-32].

We evaluated the stacking energy, $E_{\text {stack }}$ per unit cell per layer as follows:

$E_{\text {stack }}=-\left(E_{\text {layer }} / 2-E_{\text {mono }}\right)$,

where $E_{\text {mono }}$ and $E_{\text {layer }}$ are the total Mermin free energies of the optimized monolayer and the optimized two layers in the unit cells in each symmetry, respectively. $E_{\text {layer }}$ should be divided by two due to the use of two explicit layers in one unit cell.

\subsection{Rotation of FPBA p-phenylene rings in monolayer and stacked models}

We computed rotational barriers of the FPBA $p$-phenylene groups surrounding the $\mathrm{ZnP}$ units to understand the influence of this rotation on the energetics of particular stacking orders. Since these calculations are computationally more demanding, we constructed hydrogen-terminated molecular models of the COF. In order to investigate the size dependency of the molecular model we employed one small and one large model as shown in Fig. $5(b, c)$. We estimated the rotational barrier by carrying out relaxed scans of the isolated monolayer models by changing the dihedral angle, $\theta$, between the rotating $p$-phenylene group and the porphyrin ring as shown in Fig. S3. We also performed single point calculations using PBE-D2 based on the optimized structures by DFTB at each angle to compare the 
vibrational rotational angles between DFTB and DFT results. Following the evaluation of $p$-phenylene rotation in the monolayer model calculations, we investigated the effect of neighbor layers on the rotational barrier in the molecular models. We employed a three-layered model and fixed terminal carbon and nitrogen atoms as shown in Fig. S3 during optimization to reproduce the rigid crystal environment, using small and large models.

\section{Results and Discussion}

\subsection{Structural and energetic evaluation of monolayer structures}

The relative energies of each form optimized by DFTB and the Boltzmann distributions at $393 \mathrm{~K}\left(120^{\circ} \mathrm{C}\right.$, corresponding to experimental synthesis conditions) are summarized in Table 1. The structures of $\mathbf{S}_{-} S_{4}$ are the least stable using both DFTB and DFT, while the structure of $\mathbf{R} \_S_{4}$ and $\mathbf{S} \_C_{4}$ are the most stable using DFTB and DFT results respectively. However, the relative energies are distributed within a narrow window of only $3 \mathrm{kcal} / \mathrm{mol}$ in both methods, which is less than $2 \%$ of the stacking energy. The inherent accuracy of DFT is not greater than $3 \mathrm{kcal} / \mathrm{mol}$ and hence it is difficult to draw quantitative conclusions from first principles calculations. The Boltzmann distribution is however sensitive to such small energy differences and shows that some structures compete in abundance with the most stable

structure in each method. Our results suggest the possibility that under experimental conditions a variety of monolayer isomer structures can be formed, causing different local 
stacking orders during the self-assembly process of the 3D global structure. This may also be the reason for the difficulty to prepare single crystals of COFs with sufficiently large size to obtain X-ray crystal structures.

\subsection{Structural and energetic evaluation of stacked structures}

Various structures of the COF were provided by not only the diversity of local stuructures such as the rotational conformations of the $p$-phenylene groups mentioned above but also global structures such as layered manner of the each monolayer. Different COF structures can be obtained considering not only the stacking arrangement of individual monolayers, but also different local rotational conformations of the $p$-phenylene groups within each monolayer. All optimized stacked $3 \mathrm{D}$ structures of the $\mathbf{S}$ isomers and their $E_{\text {stack }}$ are listed in Table S1.

We first address the dependency of the initial slipping distances on the optimized structures. Optimizations of I and SR stacking order with initial slipping distances of 1.0, 1.5 and $2.0 \AA$ were carried out. While inclined structure I stacks remained inclined during geometry optimization independent on the different initial slipping distance, some of the serrated SR structures changed stacking order during geometry optimizations. For instance, when the initial slipping distance is $1.0 \AA$, SR type structures tend to change to $\mathbf{E}$ type structures during optimization, while optimization with an initial distance of $2.0 \AA$ tends to maintain SR type. For an initial distance of $1.5 \AA$, the optimized structure becomes $\mathbf{E}$ or SR 
types. We focused on the initial distance of $1.5 \AA$ for the optimization of the $\mathbf{R}$ isomers, as an analogous case of the $\mathbf{S}$ isomers. The optimized stacked structures of the $\mathbf{R}$ isomers and their $E_{\text {stack }}$ are listed in Table S2. The most stable structure, $\mathbf{S} C_{2}$-I-xy showed the largest $E_{\text {stack, }}, 140.1 \mathrm{kcal} / \mathrm{mol}$ among those considered in this work. We selected this overall most stable structure among all optimized 3D structures and for simplicity labeled it 1. In order to reveal contributions from components of the layer to the stacking energies, we employed a finite model as shown in Fig. S2, and we divided it into small fragments. We employed dimer models of the fragments terminated with hydrogen (i.e. CuPc-BA, FP1-4, ZnP_ring, Ph1_4, $\mathrm{CuPc}-\mathrm{FPBA}$, and $\mathrm{ZnP}-\mathrm{Ph}$ ), and evaluated their stabilization energies at the optimized geometry of 1 . The stacking energy of each component, $E_{\mathrm{s}_{-} \mathrm{cmpt}}$, was defined as follows:

$$
E_{\mathrm{s}_{-} \mathrm{cmpt}}=-\left(E_{\mathrm{dimer}}-E_{\mathrm{mono1}}-E_{\mathrm{mono2} 2}\right)
$$

where $E_{\text {dimer }}$ is the single-point energy of the 2-layer model of each component and $E_{\text {monol }}$ and $E_{\text {mono2 }}$ are the single-point energies of each component, respectively, evaluated at the geometries obtained in crystal structure geometry optimizations.

Each pair of the components in the adjacent layers stacked within the distance of 3.2 to 3.6 ̊. $E_{\mathrm{S}_{-} \mathrm{cmpt}}$ of each component of $\mathbf{1}$ is shown in Table S3. Larger components such as CuPc-BA tend to show larger stabilization. These stacking stabilizations made $\mathbf{1}$ the most stable structure with largest $E_{\text {stack, }}$ though the monolayer of $\mathbf{1}$ was less stable than the optimized S_ $C_{2}$ monolayer by $12.1 \mathrm{kcal} / \mathrm{mol}$. The averaged rotational dihedral angle of the 
four $p$-phenylene groups around $\mathrm{ZnP}$ in $\mathbf{1}$ is $50.6^{\circ}$, which is smaller than in the $\mathbf{S} C_{2}$ monolayer, $70.1^{\circ}$. As shown in Table S3 the cell parameters of $\mathbf{1}$ are $\alpha<90^{\circ}$ and $\beta<90^{\circ}$ because of the inclination of $\mathbf{1}$ in xy direction, which means that $\mathbf{1}$ is not perfectly rectangular but actually belongs to the triclinic crystal systems. As shown in Fig. 4(a), the simulated XRD pattern of 1 has a peak with the largest intensity around $2 \theta=3.6 \sim 3.7^{\circ}$, assigned to $(1 \overline{1} 0)$, at a similar position to the experimental one. However, the simulated XRD of $\mathbf{1}$ is inconsistent with experiment due to the additional peak around $2 \theta=4.0^{\circ}$, assigned to (110). We found that some structures of the $\mathbf{R}$ isomers showed $E_{\text {stack }}$ comparable to that of $\mathbf{1}$. For instance, the most stable $\mathbf{R}$ form structure, $\mathbf{R} \_C_{2}-\mathbf{I}-\mathbf{x y}$, showed a slightly smaller $E_{\text {stack }}$ than $\mathbf{1}$ $(138.0 \mathrm{kcal} / \mathrm{mol})$, and the XRD pattern of this structure also disagreed with experiment, as shown in Fig. S4. Subsequently, we simulated the XRD patterns of all optimized stacked 3D structures, and selected the structures that have only one peak consistent with experimental XRD data. The structure with the largest $E_{\text {stack }}$ among them is $\mathbf{S} \_S_{4}-\mathbf{E}$ (2) with an $E_{\text {stack }}$ of $132.1 \mathrm{kcal} / \mathrm{mol}$, which is $8.5 \mathrm{kcal} / \mathrm{mol}$ smaller relative to 1 . The $E_{\mathrm{S}_{-} \mathrm{cmpt}}$ of each component in $\mathbf{2}$ is shown in Table S4. Compared to $\mathbf{1}$, the $E_{\mathrm{s}_{-} \text {cmpt }}$ of the small components of $\mathbf{2}$ are similar or larger, while $E_{\mathrm{s}_{-} \text {cmpt }}$ of the combined components of $\mathbf{2}$ are smaller. We supposed that the stabilizations of each component with lateral components in $\mathbf{1}$ are larger than those in $\mathbf{2}$. The averaged rotational dihedral angle of the $p$-phenylene groups around $\mathrm{ZnP}$ in 2 is $41.5^{\circ}$, which is smaller than that observed in $\mathbf{1}$. The simulated XRD pattern of 2 is shown in Fig. 4(b), 
which agrees with experiment. As shown in Table S3, the cell parameters of 2 are $\alpha=90.0^{\circ}$, $\beta=90.2^{\circ}, \gamma=90.0^{\circ}$ and $a=b=34.4 \AA$, which imply that 2 can be categorized as a tetragonal

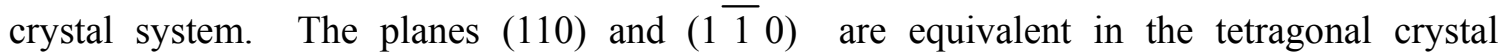
system. As summarized in Table $\mathrm{S} 1$ and Table $\mathrm{S} 2$, the $E_{\text {stack }}$ for the I type structures, including 1, tend to show higher stability and larger $E_{\text {stack, }}$ though they disagree with the experimental XRD pattern. We propose that inclination between monolayers reduces the steric repulsion between them, in particular between the twisted $p$-phenylene groups around ZnP. The conformational relaxation with smaller steric hindrance in the monolayer of I leads to larger $E_{\text {stack }}$ compared to $\mathbf{E}, \mathbf{S R}$, and SG. However, the stabilization in I results is purely enthalpic in nature, since it is obtained from optimized geometries and does not include thermal (entropic) effects. In experimental $\mathrm{COF}$ synthesis at $120^{\circ} \mathrm{C}$, thermal structural fluctuation such as rotation of the $p$-phenylene group around $\mathrm{ZnP}$ are expected to have significant influence on the stacking order, because the range of relative energies of the monolayers with different phenylene conformations was so small that various structures can be formed easily.

\subsection{Rotation of FPBA p-phenylene groups}

According to our discussion regarding the conformation of $p$-phenylene rings around $\mathrm{ZnP}$, their rotation seems to play an important role in the formation of the stacked structures of the COF. We calculated the rotational barrier along the dihedral angle $\theta$ to reveal the 
relationship between the rotational dynamics and the stacking order of the $3 \mathrm{D}$ structure. $\mathrm{We}$ defined $\theta=0^{\circ}$ for $\mathbf{1}$, which corresponds to a dihedral angle of $49.7^{\circ}$ between the planes of the p-phenylene and the $\mathrm{ZnP}$ units. As shown in Fig. 5(a), the energy curves of smaller and larger monolayer models show similar rotational barriers and profiles, indicating that the smaller model is sufficiently large to discuss the rotational barrier in the monolayer case. The rotational barrier of the monolayer model calculated by DFTB, $12 \mathrm{kcal} / \mathrm{mol}$ shown in Fig. 5(a), indicates that the $p$-phenylene groups can completely rotate at room temperature, with wide minima around $40^{\circ}$ and $219^{\circ}$, where the rotating $p$-phenylene groups and the porphyrin ring are almost diagonal. Large vibrational amplitudes are feasible in these potential energy wells. The DFT single point energies calculated at DFTB-optimized geometries, shown in Fig. S6, show a similar profile to that predicted by DFTB, validating its use for the calculation of rotational barriers in stacked molecular models.

For this purpose, we employed 3-layered isolated models and rotated a $p$-phenylene groups in the middle layer as described above. As shown in Fig. 5(a), the energy curves of smaller and larger models show similar profiles, except for a discrepancy at $\theta=120^{\circ}$, which is discussed in the SI. The rotational barrier in the smaller isolated model is $25 \mathrm{kcal} / \mathrm{mol}$, which is larger than in the monolayer model, and it is certainly not feasible to be overcome at room temperature. The minimal energy in the layered model was obtained at $\theta=0^{\circ}$. For the layered model the $p$-phenylene groups can rotate $\sim 34^{\circ}$ from $-10^{\circ}$ to $24^{\circ}$ and from $173^{\circ}$ to $207^{\circ}$, 
which is much smaller than the range observed in the monolayer model, $\sim 74^{\circ}$ from $2^{\circ}$ to $77^{\circ}$

and from $182^{\circ}$ to $256^{\circ}$. These differences are caused by the steric repulsion between the rotating $p$-phenylene groups and the adjacent layers, which restricts rotation to a narrower range and increases the rotational barrier in the layered model. The restriction of rotation can prevent the formation of the thermodynamically most favorable structure, leading to the formation of layered structures with an overall SR type, or more complicated stacking order, as a result.

\section{Concluding remarks}

We theoretically investigated the structural diversity of a recently synthesized double-stage COF, CuPc-FPBA-ZnP. This COF has three units to form a large variety of local structures, in addition to different stacking orders and rotational vibration of linkers. First, we quantum chemically optimized isomeric monolayers of CuPc-FPBA-ZnP and showed the competitiveness of various isomers, which already indicates that formation of a well-ordered single crystal must be difficult, as observed experimentally. Second, we optimized stacked structures based on the optimized monolayer structures. We found that an inclined I type structure, $\mathbf{1}$, is most stable and possesses the largest stacking energy $E_{\text {stack. }}$. However, the simulated powder XRD pattern of 1 disagrees with the experimental results, while those of some $\mathbf{E}$ and $\mathbf{S R}$ type structures reproduced experiment well. We proposed 
that this disagreement derived from the lack of considering thermal structural fluctuation of frameworks in the optimization of the COF. This is corroborated by the shallow calculated rotational barriers of the FPBA $p$-phenylene groups around $\mathrm{ZnP}$ in the monolayers as opposed to steep potential energy wells separated by high rotational barriers after stacking. We concluded that the $p$-phenylene rotation during layer formation generates various isomers. As a result of small discrepancies in the relative energy among the isomers, various other conformational isomers are competing with the global minimum energy structure and influence the formation processes of COFs. We therefore conclude that in order to obtain COFs with higher crystallinity, freely rotatable groups such as $p$-phenylene groups should be avoided.

\section{Acknowledgements}

This work was supported by a JSPS Open Partnership grant (13039901-000174) for bilateral researcher exchange and by a CREST (Core Research for Evolutional Science and Technology) grant to SI from JST. 


\section{References}

[1] M.E. Davis, Nature 417 (2002) 813.

[2] S. Kitagawa, R. Kitaura, S. Noro, Angew. Chem. Int. Ed. 43 (2004) 2334.

[3] A.P. Côté, A.I. Benin, N.W. Ockwig, M. O'Keeffe, A.J. Matzger, O.M. Yaghi, Science 310 (2005) 1166.

[4] X. Feng, X. Ding, D. Jiang, Chem. Soc. Rev. 41 (2012) 6010.

[5] P.J. Waller, F. Gandara, O.M. Yaghi, Acc. Chem. Res. 48 (2015) 3053.

[6] S. Chandra, T. Kundu, S. Kandambeth, R. Babarao, Y. Marathe, S.M. Kunjir, R. Banerjee, J. Am. Chem. Soc. 136 (2014) 6570.

[7] A.P. Côté, H.M. El-Kaderi, H. Furukawa, J.R. Hunt, O.M. Yaghi, J. Am. Chem. Soc. 129 (2007) 12914.

[8] H. Furukawa, O.M. Yaghi, J. Am. Chem. Soc. 131 (2009) 8875.

[9] S.Y. Ding, J. Gao, Q. Wang, Y. Zhang, W.G. Song, C.Y. Su, W. Wang, J. Am. Chem. Soc. 133 (2011) 19816.

[10] M. Dogru, T. Bein, Chem. Commun. 50 (2014) 5531.

[11] Y. Du, H. Yang, J.M. Whiteley, S. Wan, Y. Jin, S.-H. Lee, W. Zhang, Angew. Chem. Int. Ed. 55 (2016) 1737.

[12] D.N. Bunck, W.R. Dichtel, Angew. Chem. Int. Ed. 51 (2012) 1885.

[13] S. Jin, M. Supur, M. Addicoat, K. Furukawa, L. Chen, T. Nakamura, S. Fukuzumi, S. Irle, D. Jiang, J. Am. Chem. Soc. 137 (2015) 7817.

[14] B. Lukose, A. Kuc, J. Frenzel, T. Heine, Beilstein J. Nanotechnol. 1 (2010) 60.

[15] Z. Hu, L. Zhang, J. Jiang, J. Chem. Phys. 136 (2012) 244703.

[16] Y.B. Zhang, J. Su, H. Furukawa, Y. Yun, F. Gandara, A. Duong, X. Zou, O.M. Yaghi, J. Am. Chem. Soc. 135 (2013) 16336.

[17] D. Beaudoin, T. Maris, J.D. Wuest, Nat. Chem. 5 (2013) 830.

[18] J. Cravillon, R. Nayuk, S. Springer, A. Feldhoff, K. Huber, M. Wiebcke, Chem. Mater. 23 (2011) 2130.

[19] L. Ascherl, T. Sick, J.T. Margraf, S.H. Lapidus, M. Calik, C. Hettstedt, K. Karaghiosoff, M. Döblinger, T. Clark, K.W. Chapman, F. Auras, T. Bein, Nat. Chem. 8 (2016) 310.

[20] H. Rietveld, Acta Crystallogr. 22 (1967) 151.

[21] M.G. Rabbani, A.K. Sekizkardes, Z. Kahveci, T.E. Reich, R. Ding, H.M. El-Kaderi, Chem. Eur. J. 19 (2013) 3324.

[22] L.B. McCusker, R.B. Von Dreele, D.E. Cox, D. Louer, P. Scardi, J. Appl. Cryst. 32 (1999) 36.

[23] M. Inukai, S. Horike, D. Umeyama, Y. Hijikata, S. Kitagawa, Dalton Trans. 41 (2012) 13261.

[24] X. Chen, M. Addicoat, E. Jin, H. Xu, T. Hayashi, F. Xu, N. Huang, S. Irle, D. Jiang, Sci. Rep. 5 (2015) 14650. 
[25] M. Elstner, D. Porezag, G. Jungnickel, J. Elsner, M. Haugk, T. Frauenheim, S. Suhai, G. Seifert, Phys. Rev. B 58 (1998) 7260.

[26] A.K. Rappe, C.J. Casewit, K.S. Colwell, W.A. Goddard, W.M. Skiff, J. Am. Chem. Soc. 114 (1992) 10024.

[27] J.P. Perdew, K. Burke, M. Ernzerhof, Phys. Rev. Lett. 77 (1996) 3865.

[28] S. Grimme, J. Comput. Chem. 27 (2006) 1787.

[29] R. Taylor, C.F. Macrae, Acta Crystallogr. B 57 (2001) 815.

[30] I.J. Bruno, J.C. Cole, P.R. Edgington, M. Kessler, C.F. Macrae, P. McCabe, J. Pearson, R. Taylor, Acta Crystallogr. B 58 (2002) 389.

[31] C.F. Macrae, P.R. Edgington, P. McCabe, E. Pidcock, G.P. Shields, R. Taylor, M. Towler, J. van de Streek, J. Appl. Cryst. 39 (2006) 453.

[32] C.F. Macrae, I.J. Bruno, J.A. Chisholm, P.R. Edgington, P. McCabe, E. Pidcock, L. Rodriguez-Monge, R. Taylor, J. van de Streek, P.A. Wood, J. Appl. Cryst. 41 (2008) 466. 

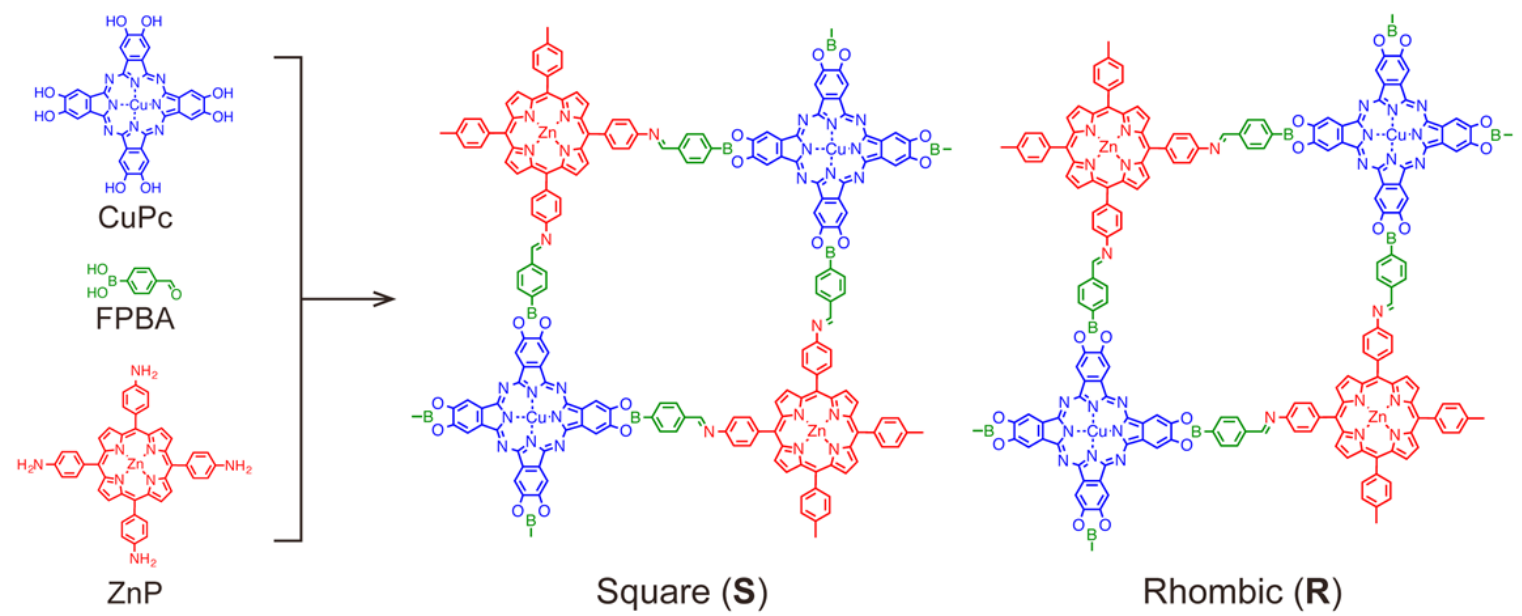

CuPc-FPBA-ZnP

Fig. 1. Schematic structures of monomers, $\mathrm{ZnP}$ (red), CuPc (blue) and FPBA (green), and S and $\mathbf{R}$ isomers of a CuPc-FPBA-ZnP monolayer.
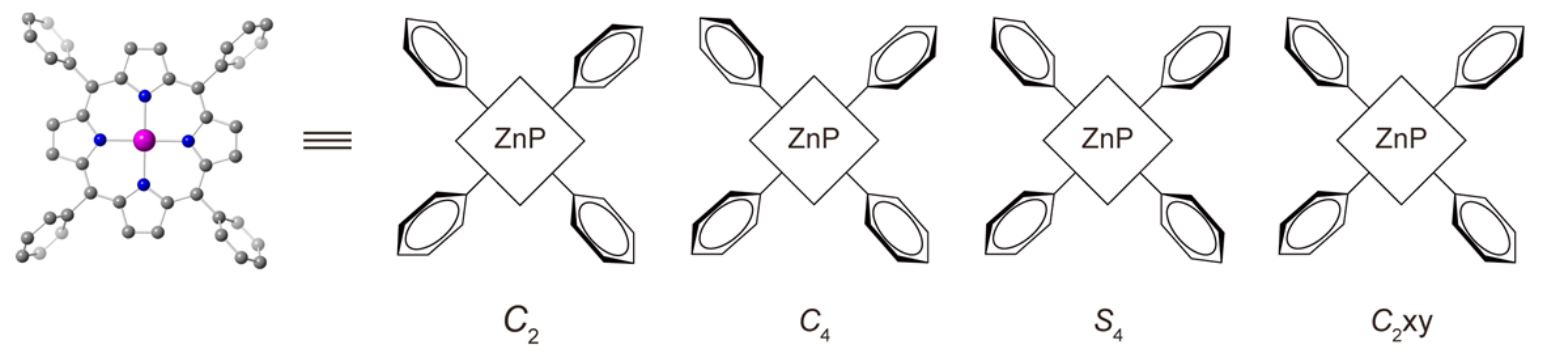

Fig. 2. Rotational patterns of the four phenyl rings of the FPBA unit connected to a $\mathrm{ZnP}$ unit. The gray, blue, and magenta spheres are $\mathrm{C}, \mathrm{N}$, and $\mathrm{Zn}$, respectively. $\mathrm{H}$ atoms are omitted for clarity. 


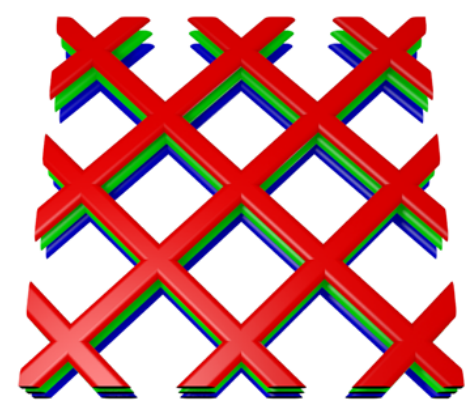

Eclipsed (E)

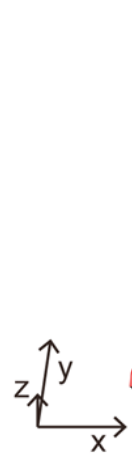

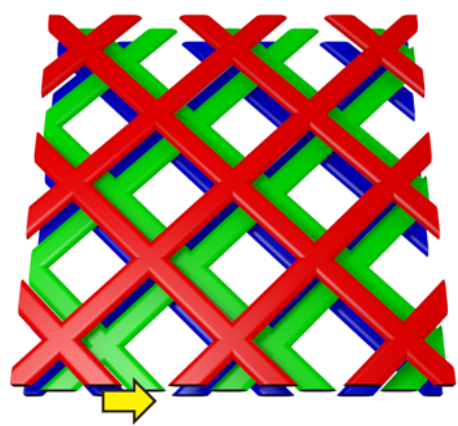

Serrated x (SR-x)

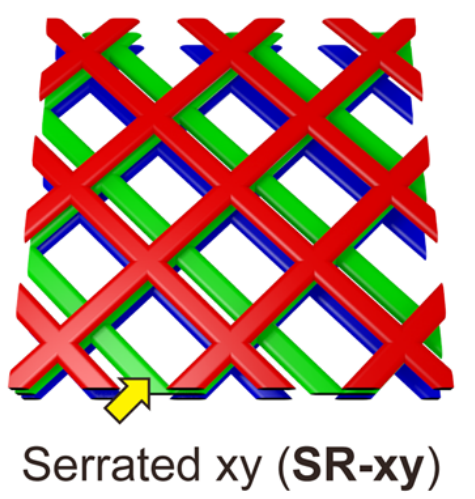

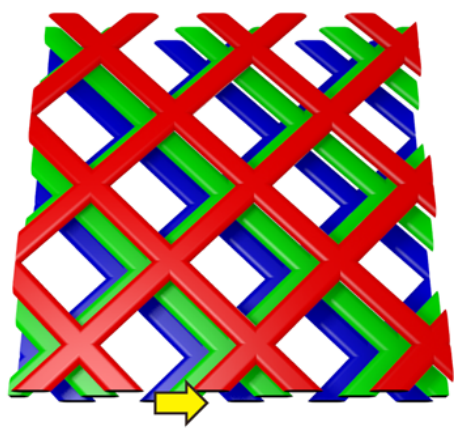

Inclined x (I-x)

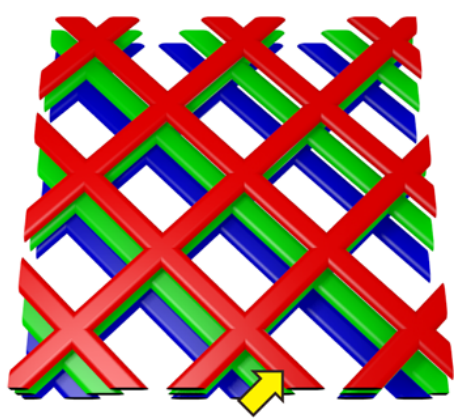

Inclined xy (I-xy)

Fig. 3. Schematic stacking order of the monolayers: Eclipsed (E), staggered (SG), serrated x

(SR-x), serrated xy (SR-xy), inclined x (I-x), and inclined xy (I-xy). SR-y and I-y are omitted because of their similarity to SR-x and I-x, respectively. Yellow arrows indicate the slipping directions. We depicted three layers to show the stacking manner, although we employed two layers in a unit cell and blue and red layers are identical. 
(a)
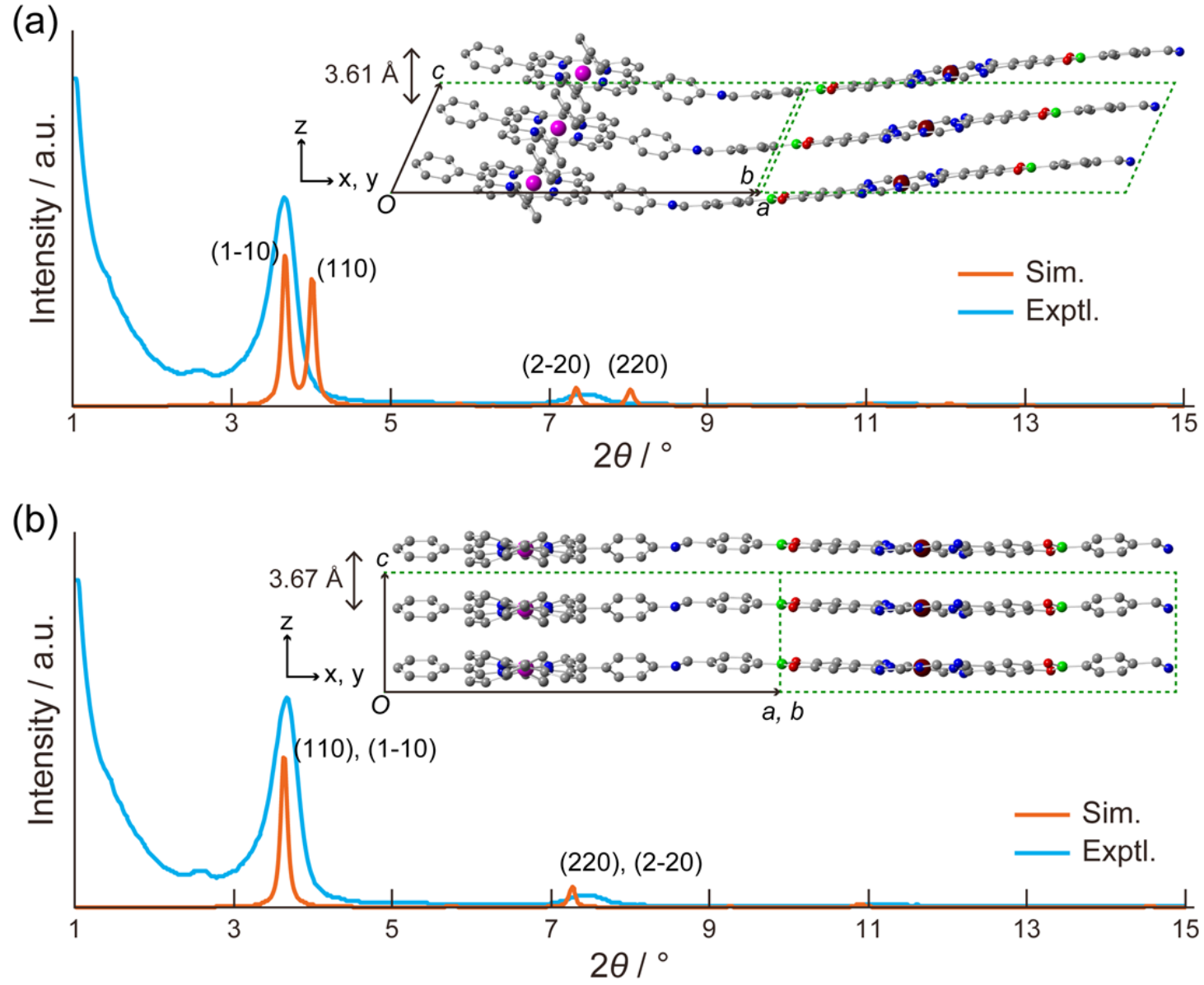

Fig. 4. (a) The simulated XRD pattern of $\mathbf{1}$ (orange) and the experimental XRD pattern (cyan).

Side view of $\mathbf{1}$ is inserted. (b) The simulated XRD pattern of $\mathbf{2}$ (orange) and the experimental XRD pattern (cyan). Side view of $\mathbf{2}$ is inserted. Three layers are used to show how to stack for each side view, though only two layers are put in a unit cell. 


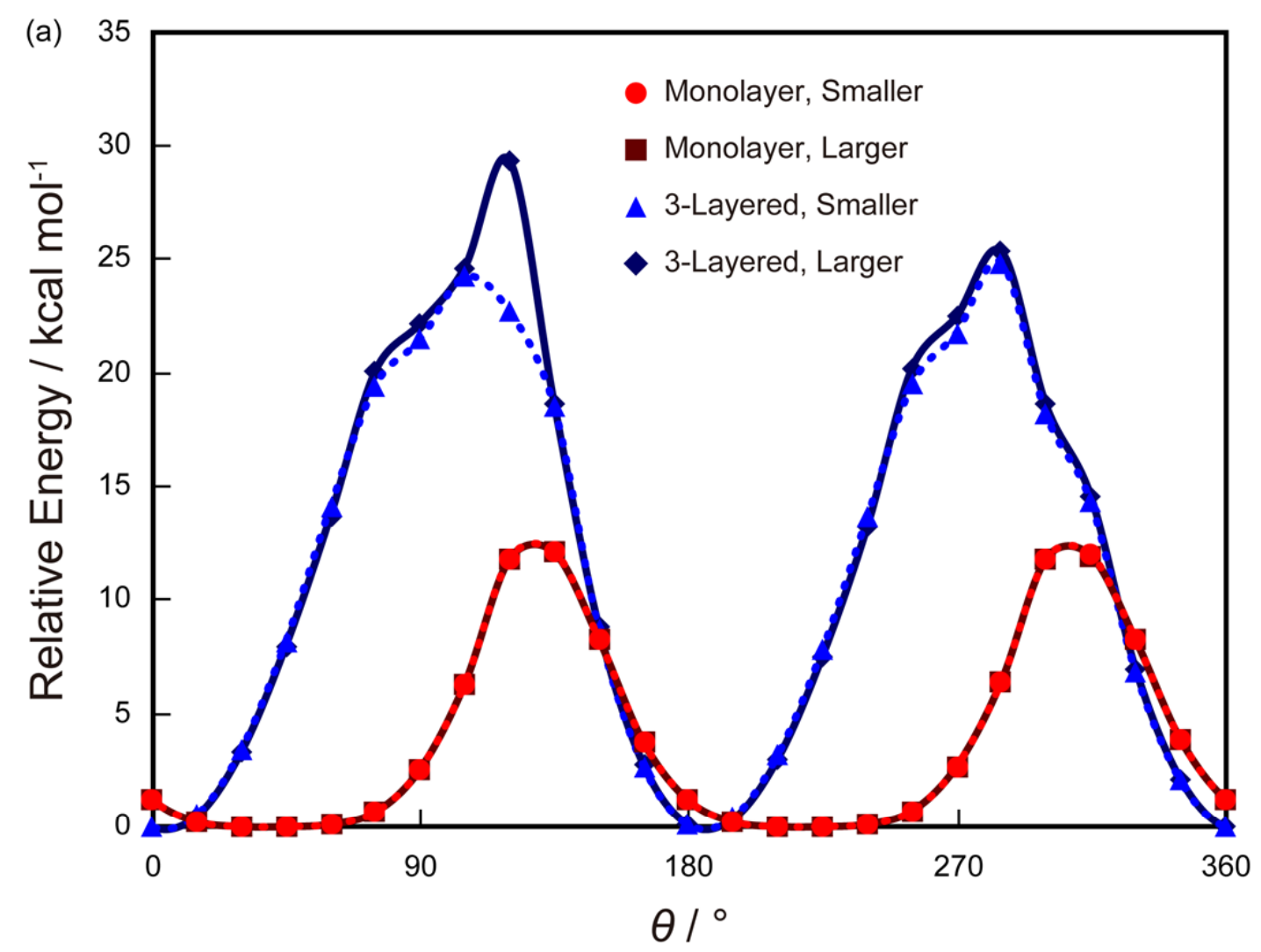

(b)

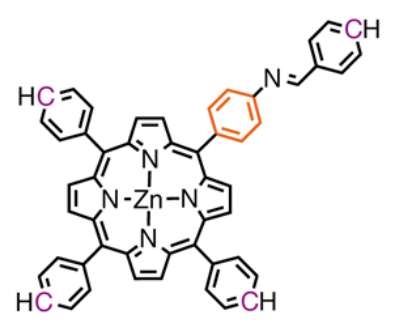

Smaller (c)<smiles>Nc1ccc(Br)cc1</smiles> 
groups indicate the rotating rings. Terminal carbon and nitrogen atoms colored by purple color indicate the fixed atoms in all 3 layers during the relaxation of three layered models.

\section{Table 1}

DFTB and DFT relative energies of monolayer structures of CuPc-FPBA-ZnP after DFTB geometry optimization in $\mathrm{kcal} / \mathrm{mol}$. Boltzmann distributions at $393 \mathrm{~K}$ are shown in parentheses.

\begin{tabular}{|c|c|c|c|c|c|c|c|c|}
\hline Method & $\mathbf{S} \_C_{2}$ & S_C $C_{4}$ & S_ $S_{4}$ & $\mathrm{~S} \_C_{2} \mathrm{xy}$ & $\mathbf{R} \_C_{2}$ & $\mathbf{R} \_C_{4}$ & $\mathbf{R} \_S_{4}$ & $\mathbf{R} \_C_{2} \mathrm{xy}$ \\
\hline \multirow[t]{2}{*}{ DFTB } & 1.73 & 0.89 & 2.20 & 1.53 & 0.96 & 1.35 & 0.00 & 0.64 \\
\hline & $(4 \%)$ & $(14 \%)$ & $(2 \%)$ & $(6 \%)$ & $(11 \%)$ & $(7 \%)$ & $(38 \%)$ & $(17 \%)$ \\
\hline \multirow[t]{2}{*}{ DFT } & 1.07 & 0.00 & 2.94 & 1.46 & 0.27 & 0.78 & 1.56 & 1.97 \\
\hline & $(9 \%)$ & $(37 \%)$ & $(1 \%)$ & $(6 \%)$ & $(26 \%)$ & $(14 \%)$ & $(5 \%)$ & $(3 \%)$ \\
\hline
\end{tabular}


Table of Contents Figure
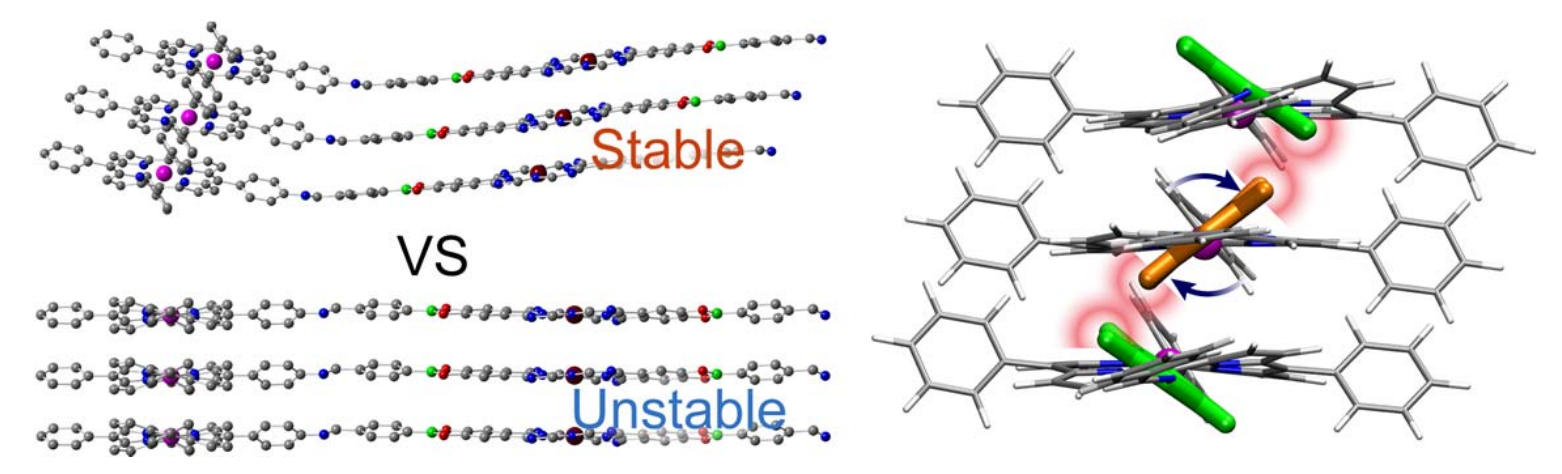